\title{
SUMMARY
}

CAVALCANTI, Sarah Desirée Barbosa - Aplicação de metodologias de preservação e caracterização de fungos na coleção de culturas do Instituto de Medicina Tropical de São Paulo. [Dissertação]. São Paulo, Faculdade de Medicina da Universidade de São Paulo, 2010.

\section{APPLICATION OF PRESERVATION METHODS AND CHARACTERIZATION OF FUNGAL ISOLATES FROM THE INSTITUTO DE MEDICINA TROPICAL DE SÃO PAULO CULTURE COLLECTION}

The fungal culture collection from the Instituto de Medicina Tropical de São Paulo/USP comprises 1047 isolates of fungi, algae and actinomycetes with medical and veterinary relevance, which up to now have been preserved by subculturing every three months. Created in 1925 by Prof. Floriano Paulo de Almeida and maintained by Prof. Carlos da Silva Lacaz and his assistants since 1953, this fungal collection needed to be realocated to another area in 2007, as well as updated and revised as to its preservation methods. This new area was reformed according to safety procedures recommended in guidelines for pathogenic microorganisms manipulation. Several studies demonstrated that storage of fungi by subculturing may induce antigenic changes, phenotypic and genetic alterations, in addition to attenuation of virulence. The present study was aimed at utilizing more adequate methods of long-term preservation, such as lyophilization, sterile distilled water, and cryopreservation (freezing at $-80{ }^{\circ} \mathrm{C}$ and storage in liquid nitrogen $\left[-196^{\circ} \mathrm{C}\right]$ ). Three hundred eighty six isolates consisting of dimorphic (213) and filamentous fungi (hyaline and melanized - 106), and yeasts (67) associated with the most prevalent fungal diseases in our setting were randomly selected. Two methods were applied for storage of each fungal group: yeasts were submitted to lyophilization and freezing at $-80{ }^{\circ} \mathrm{C}$; dimorphic and filamentous fungi were preserved in sterile distilled water and liquid nitrogen. All isolates were initially evaluated for their morphological features, submitted to the different methods of storage, and during a period of up to two years, reassessed for viability and morphological features. Yeasts showed $100 \%$ viability by the two applied preservation methods, maintaining their phenotypic characteristics. Similarly, the two methods applied to filamentous fungi resulted in high rates of viability, with maintenance of morphology. Regarding the dimorphic fungi, the preservation in liquid nitrogen resulted in variable rates of recovery, depending on the genus assessed. From all studied isolates, 75 were also submitted to molecular identification by sequencing after PCR amplification of the ITS region of the ribossomal DNA. This molecular technique showed, for some isolates, a lack of agreement in species identification when compared with the phenotypic methods. The new long-term storage methods used in the study preserved the typical genus and species morphology of the studied isolates, while resulting in high viability rates. The molecular identification proved to be an important tool for the authentication of isolates.

Sarah Desirée Barbosa Cavalcanti sarahdbc@usp.br 